\title{
Ischemia and activated neutrophils alter coronary microvascular but not epicardial coronary artery reactivity
}

Activated neutrophils have been implicated in reperfusion injury and the no-reflow phenomenon of intramyocardial arterioles. This study tested the hypothesis that ischemia and activated neutrophils impair coronary endothelial and smooth muscle cell function of epicardial and intramyocardial coronary arteries. Alteration of smooth muscle and endothelial cell function in epicardial coronary arteries ( $3 \mathrm{~mm}$ diameter) and intramyocardial coronary arteries $(0.3 \mathrm{~mm}$ diameter) was compared by means of a myograph after exposure to ischemia (epicardial, 160 minutes; intramyocardial, 30 minutes), activated neutrophils, and combined ischemia and activated neutrophils. Morphologic studies at the ultrastructural level were done by means of scanning electron microscopy. Epicardial coronary artery function was normal after ischemia, storage with activated neutrophils, and ischemia followed by storage with activated neutrophils. Intramyocardial artery function, however, was altered. Contraction to a $45 \mathrm{mmol} / \mathrm{L}$ concentration of potassium chloride after ischemia and storage with activated neutrophils was increased $(p=0.06)$. Smooth muscle relaxation was significantly decreased after ischemia, but storage with activated neutrophils did not further decrease smooth muscle relaxation. Endothelium-dependent relaxation to bradykinin was significantly decreased after combined ischemia and incubation with activated neutrophils $(p<0.05)$. Sensitivity to bradykinin was decreased after both ischemia alone $(p<0.05)$ and activated neutrophils alone $(p<0.05)$. Similar morphologic alterations were found in epicardial and intramyocardial arteries after ischemia. Activated neutrophils alone minimally damaged endothelial cells of nonischemic intramyocardial and epicardial arteries. Endothelial cells of both arteries exposed to ischemia alone showed evidence of ischemic damage, including endothelial cell blebbing, nuclear bulging, and appearance of large holes in the cell surface. Severe endothelial cell damage was found after combined ischemia and storage with neutrophils: total destruction of cells and exposure of the basal lamina. Endothelial damage, therefore, correlated with artery function in intramyocardial but not in epicardial arteries. These results indicate that ischemia is a prerequisite for severe neutrophil injury of intramyocardial artery endothelium-mediated relaxation. This may explain no-reflow phenomenon in arterioles concurrent with myocardial reperfusion injury. ( $J$ Thorac Cardiovasc Surg 1994;108:648-57)

Margit Kadletz, MD, Rebecca J. Dignan, MD, ${ }^{\text {a }}$ Kathryn E. Loesser, $\mathrm{PhD},{ }^{\mathrm{b}}$

Michael L. Hess, MD, ${ }^{b}$ and Andrew S. Wechsler, MD, ${ }^{a}$ Richmond, Va.

From the Departments of Surgery ${ }^{\mathbf{a}}$ and Cardiology, ${ }^{\mathrm{b}}$ Medical College of Virginia, Richmond, Va.

Supported by the Fonds zur Förderung der wissenschaftlichen Forschung J0572-MED, Austria, and by the National Heart Lung, and Blood Institute, 5 R01 HL26302-11.

Received for publication Nov. 24, 1992.

Accepted for publication April 13, 1994.

Address for reprints: Andrew S. Wechsler, MD, Department of Surgery, Medical College of Virginia, MCV Station Box 645, Richmond, VA 23298-0645.

Copyright $\odot 1994$ by Mosby-Year Book, Inc.

$0022-5223 / 94 \$ 3.00+0 \quad \mathbf{1 2} / \mathbf{1} / \mathbf{5 6 8 1 2}$
Q oronary vascular tone is influenced by circulating and locally released vasoactive substances. Since Furchgott and Zawadzki ${ }^{1}$ first described the important role of endothelial cells in regulation of vascular tone, considerable effort has been expended to investigate the physiologic function of endothelium. An abnormal endothelial state may exist after ischemia and during reperfusion. Endothelial cells damaged by ischemia and reperfusion may have diminished release of vasoactive substances such as endothelium-derived relaxing factor (EDRF). Under physiologic conditions, EDRF is a labile com- 
pound produced by endothelial cells, activated by vasoactive substances such as acetylcholine, bradykinin, histamine, substance $P$, and serotonin. Nitric oxide has been identified as the chemical mediator. Impaired release or production of EDRF may predispose arteries to vaso$\mathrm{spasm}^{2-5}$ and decrease myocardial perfusion after ischemia.

Decreased infarct size has been demonstrated by depletion of neutrophils from reperfusion blood, ${ }^{6}$ an observation that implicates neutrophils in infarction. Quillen and associates ${ }^{7}$ demonstrated altered microvessel endothelial cell function after ischemia and reperfusion with whole blood in dogs. They also noted a significant decrease in EDRF production by microvessel endothelial cells compared with epicardial vessels after ischemia and whole blood reperfusion.

It is difficult to determine, however, whether ischemia, whole blood reperfusion, or a combination is the cause of injury to endothelium. In previous studies, normal endothelium-mediated relaxation was demonstrated after 30 minutes of ischemia in intramyocardial arteries (unpublished data) and after 160 minutes of ischemia in epicardial arteries. ${ }^{8}$ Function declined significantly after 60 minutes and 180 minutes of ischemia, respectively. Reperfusion of whole blood alone or reperfusion of one of its components, therefore, may be the cause of endothelial injury during shorter periods of ischemia. To demonstrate the isolated effect of ischemia, we used an excised heart model to exclude collateral flow and to control reperfusion. We then tested whether addition of neutrophils in a crystalloid perfusate would interact with the postischemic vasculature to produce specific cell-mediated reperfusion injury. We wished to isolate the neutrophil effects from those of whole blood confounding effects such as release of platelet factors, binding of nitric oxide to heme molecules, and the possibility of erytrocyte plugging of small arteries.

The aim of this study was to determine whether ischemia alone, activated neutrophils alone, or combined ischemia with activated neutrophils cause alteration in EDRF-dependent responses of epicardial and intramyocardial arterial endothelial cells.

\section{Materials and methods}

All protocols met the National Institutes of Health guidelines for the care and use of laboratory animals and were approved by the Medical College of Virginia/Virginia Commonwealth University's Institutional Animal Care and Use Committee.

Pigs $(n=12)$ were anesthetized with ketamine hydrochloride $(22 \mathrm{mg} / \mathrm{kg})$ and pentobarbital $(30 \mathrm{mg} / \mathrm{kg})$. After tracheostomy the pigs were ventilated. After heparinization $(30 \mathrm{U} / \mathrm{kg})$, a rapid cardiectomy was performed. The beating heart was divided transversly into two pieces across the left anterior descending artery (LAD) and the circumflex artery (CX). The control piece was stored in oxygenated physiologic saline solution (PSS) at $4^{\circ} \mathrm{C}$. The second piece was stored for 30 or 160 minutes at $37^{\circ} \mathrm{C}$ in PSS without oxygen. Ischemic time wàs determined from previous studies ${ }^{8}$ (manuscript in preparation), which showed significantly decreased endothelium-mediated smooth muscle relaxation of epicardial ring segments after more than 160 minutes $^{8}$ and intramyocardial ring segments after more than 30 minutes (manuscript in preparation ). Times were chosen to maximize ischemic injury without significant decrease in function to discern the subsequent influence of neutrophils.

Isolation of neutrophils. Neutrophils were isolated by a modified Henson method. ${ }^{9}$ Heparin anticoagulated blood was mixed with dextrose in $0.9 \%$ sodium chloride (1:3). Red blood cells were allowed to settle for 30 minutes at room temperature. Supernatant rich in white blood cells was placed over a FicollHypaque gradient (Sigma Chemical Company, St. Louis, Mo.) and centrifuged at $400 \mathrm{~g}$ for 30 minutes to extract neutrophils. Remaining red cells in the resulting pellet were lysed with water for 25 seconds, and PSS was made by adding 3.9\% saline solution. After centrifugation for 10 minutes at $400 \mathrm{~g}$, the supernatant was removed. Neutrophils were resuspended in PSS, counted, and placed on ice until used. Suspensions prepared by this method contained $94 \%$ neutrophils. Viability, as determined with Trypan blue staining, was $98 \%$.

The normal concentration of neutrophils in pigs is 12 million per milliliter. ${ }^{10} \mathrm{We}$ used double this concentration to simulate the physiologic concentration in postischemic myocardium. Cells were activated with a $2 \mathrm{ng} / \mathrm{ml}$ concentration of phorbol myristate acetate (Sigma) for 2 minutes at $4^{\circ} \mathrm{C}$. This concentration of phorbol myristate acetate has been shown to activate neutrophils. ${ }^{11}$ Activated neutrophils were washed once to remove phorbol myristate acetate and resuspended in oxygenated PSS at $37^{\circ} \mathrm{C}$. Activation of neutrophils was confirmed by scanning electron microscopy.

Studies on epicardial coronary ring segments. Coronary arteries (LAD $+\mathrm{CX}$ ) from control and ischemic ( 160 minutes) myocardium were dissected free of surrounding tissue and cut into $3 \mathrm{~mm}$ ring segments. Half of the control and ischemic ring segments were incubated in oxygenated PSS at $37^{\circ} \mathrm{C}$ with activated neutrophils ( 24 million per milliliter) for 30 minutes. The other half were stored until mounted on the strain gauge in oxygenated PSS at $4^{\circ} \mathrm{C}$ without activated neutrophils to serve as a control for neutrophil injury.* Epicardial ring segments were suspended on a strain gauge in a tissue bath. The upper wire was connected to a Grass FT03D force transducer (Grass Instruments Co., Quincy, Mass); the lower wire was fixed to a micrometer (Mitutoyo, Tokyo, Japan). Force responses were recorded on a Grass oscillographic recorder. Ring segments were allowed to equilibrate for 45 minutes in the tissue bath in PSS warmed to $37^{\circ} \mathrm{C}$ and gassed with $95 \%$ oxygen and $5 \%$ carbon dioxide containing indomethacin $(10 \mu \mathrm{mol} / \mathrm{L})$ to exclude prostaglandin-mediated reactions. A normalization procedure previously described ${ }^{12}$ was used to set the ring

*Epicardial and intramyocardial ring segments incubated with oxygenated neutrophil solution were kept at $37^{\circ} \mathrm{C}$ because neutrophils are more active at normothermic than hypothermic temperatures. Control and ischemic ring segments with and without neutrophils were later kept at $37^{\circ} \mathrm{C}$ in oxygenated PSS for calibration and testing for 3 to 4 hours as were ring segments not incubated with neutrophils. Ring segments were never hypoxic. 


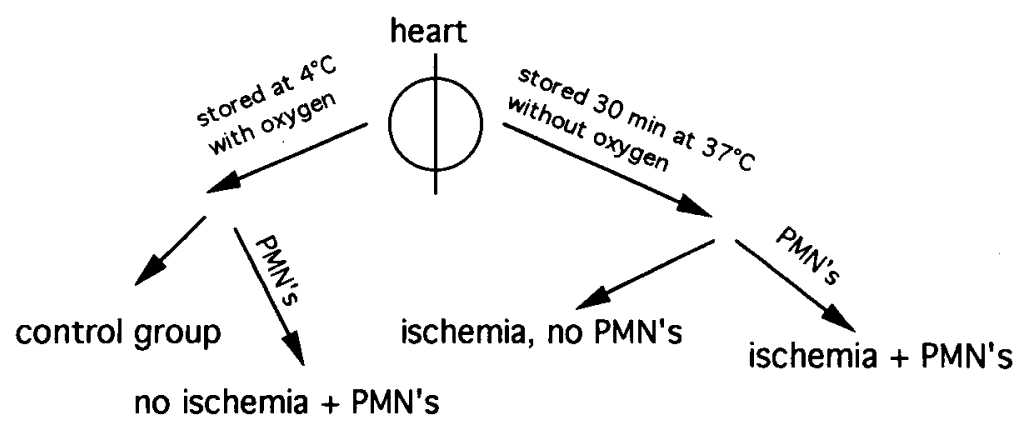

Fig. 1. Diagram of the preparation. $P M N$ 's, Polymorphonuclear leukocytes.

segments to a standard resting pressure independent of ring segment diameter. Ring segments were contracted with potassium chloride in a concentration of $125 \mathrm{mmol} / \mathrm{L}$ to obtain maximum possible contraction, a test of vascular smooth muscle integrity, and $85 \mathrm{mmol} / \mathrm{L}$ to obtain partial contraction. Increasing concentrations of bradykinin were used until no further endothelium-dependent relaxation was observed. Sodium nitroprusside, $10^{-4} \mathrm{mmol} / \mathrm{L}$, was used to produce maximum smooth muscle relaxation.*

Studies on intramyocardial coronary ring segments. The heart was cut across the LAD into two pieces. Half was stored under ischemic conditions at $37^{\circ} \mathrm{C}$, and the other half was kept at $4^{\circ} \mathrm{C}$ with oxygen to be protected against ischemia. After 30 minutes of storage, ring segments were prepared and mounted on a small vessel myograph without incubation with activated neutrophil solution. In a second step, another branch of coronary artery (LAD or CX) was prepared from an ischemic and nonischemic piece of the heart (Fig. 1). Neurophil preparation required approximately 4 hours. Both nonischemic and ischemic hearts were stored for this time at $4^{\circ} \mathrm{C}$ in oxygenated solution. The coronary artery proximal to a selected intramyocardial branch was filled gently with $5 \mathrm{ml}$ neutrophil solution ( 24 million per milliliter) with a 22-gauge catheter until all red cells were flushed from the area of dissection. This ensured filling of the intramyocardial branch with neutrophils. After incubation for 30 minutes in oxygenated PSS at $37^{\circ} \mathrm{C}$, intramyocardial arteries were removed from underlying tissue. Reactivity was tested on a microvessel myograph (University of Aarhus, Aarhus, Denmark) by attaching one wire to a pressure transducer (Dristler-Morse Corp., Bellevue, Wash.) and one wire to a micrometer (Mitutoyo). After 45 minutes of equilibration in normothermic, oxygenated ( $95 \%$ oxygen, $5 \%$ carbon dioxide), buffered saline solution containing indomethacin $10 \mu \mathrm{mol} / \mathrm{L}$, the same normalization procedure followed for the epicardial ring segments was used to set the intramyocardial ring segments to a standard resting pressure independent of ring segment diameter.

All intramyocardial ring segments were contracted twice with potassium chloride in a concentration of $125 \mathrm{mmol} / \mathrm{L}$ to obtain total possible contraction and once with $45 \mathrm{mmol} / \mathrm{L}$ to partially contract ring segments. Increasing concentrations of

*Potassium was used to precontract the ring segments because preliminary results showed that prostaglandin $\left(\mathrm{PGF}_{2 \alpha}\right)$ and thromboxane did not produce constant contractions in control intramyocardial ring segments. Alpha-receptor agents were not used because they do not contract porcine coronary arteries. bradykinin were used until no further endothelium-dependent relaxation was obtained.* Sodium nitroprusside $\left(10^{-4} \mathrm{~mol} / \mathrm{L}\right)$ was used to produce maximum smooth muscle relaxation.

Electron microscopic studies. Arteries from four to six pigs in each of the treatment groups were collected for scanning microscopy (both epicardial and intramyocardial). Control arteries and arteries after ischemia or incubation with activated neutrophils were placed in $3 \%$ glutaraldehyde in sodium phosphate buffer solution, $0.1 \mathrm{~mol} / \mathrm{L}$.

Fixed arteries were processed for scanning electron microscopy according to conventional methods. ${ }^{13}$ Samples were dehydrated in a graduated series of acetone, cut in half lengthwise, and critical-point dried, the acetone being replaced with carbon dioxide. Specimens were mounted on metal stubs, gold sputtercoated, and photographed on a JEOL SEM 100 scanning electron microscope (JEOL USA, Inc., Peabody, Mass.). Microscopic evaluation was done without knowledge of the treatment group. The entire surface of each artery was scanned at low magnification $(400 \times)$ and overall impression of damage was recorded. The entire surface could be examined because the tilt mechanism on the microscope allowed visualization of curved walls. Areas that showed damage were then examined at higher magnification $(2000 \times)$ for type of damage to the endothelial cell and exposure of underlying basal lamina. Photographs were taken of areas that were representative of the artery. Anything unusual was also photographed. Cut ends of the arteries were not evaluated because of the extensive mechanical damage. Micrographs were selected to be representative of the specimen and were given an overall score for ischemic and neutrophil damage. Data are cumulative. The microscopist was blinded to treatment groups at the time of grading.

Data analysis. Four groups of epicardial arteries were compared:

Group 1: Control ring segments without ischemia or incubation with activated neutrophils

Group 2: Ring segments not subject to ischemia but incubated with activated neutrophils, 24 million per milliliter

Group 3: Ring segments after 160 minutes of ischemia without incubation with activated neutrophils

Group 4: Ring segments after combined 160 minutes of ischemia and incubation with activated neutrophils, 24 million per milliliter

${ }^{*}$ Acetylcholine and calcium ionophore did not produce consistent endothelium-mediated relaxation in control intramyocardial arteries and, therefore, were not used. 
Table I. Internal diameter (at $100 \mathrm{~mm} \mathrm{Hg}$ ) and normalized resting pressure of epicardial coronary arteries

\begin{tabular}{lccc}
\multicolumn{1}{c}{ Groups } & $\begin{array}{c}\text { No. of } \\
\text { pigs }\end{array}$ & $\begin{array}{c}\text { ID } \\
(\mathrm{mm})\end{array}$ & $\begin{array}{c}\text { Baseline resting } \\
\text { pressure }(\mathrm{mm} \mathrm{Hg})\end{array}$ \\
\hline I (0), PMNs (0) & 11 & $3.1 \pm 0.2$ & $57.9 \pm 12.1$ \\
I (0), PMNs (24 million) & 5 & $2.8 \pm 0.2$ & $60.1 \pm 13.5$ \\
I (160), PMNs (0) & 7 & $3.1 \pm 0.2$ & $55.4 \pm 11.8$ \\
I (160), PMNs (24 million) & 5 & $2.8 \pm 0.2$ & $72.9 \pm 25.4$ \\
\hline
\end{tabular}

$I D$, Internal diameter; $I$, time of ischemia in minutes; $P M N s$, number of activated neutrophils per milliliter.

$p=$ Not significant.

Table II. Reactivity of epicardial coronary arteries

\begin{tabular}{|c|c|c|c|c|c|}
\hline Groups & $\begin{array}{c}\mathrm{KCl}(125 \mathrm{mmol} / \mathrm{L}) \\
(\mathrm{mm} \mathrm{Hg})\end{array}$ & $\begin{array}{c}\mathrm{KCl}(85 \mathrm{mmol} / \mathrm{L}) \\
(\mathrm{mm} \mathrm{Hg})\end{array}$ & $\begin{array}{c}\text { Relaxation } \\
\text { to } B K N^{*}(\%)\end{array}$ & $\begin{array}{l}E C_{50} B K N \\
(\mathrm{mmol} / \mathrm{L})\end{array}$ & $\begin{array}{c}\text { Total } S M^{+} \\
\text {relaxation }(\%)\end{array}$ \\
\hline I (0), PMNs (0) & $82.1 \pm 8.8$ & $73.1 \pm 7.0$ & $67.2 \pm 7.3$ & $6.6 \pm 4.6 \times 10^{-8}$ & $48.6 \pm 6.4$ \\
\hline I (0), PMNs (24 million) & $96.6 \pm 9.6$ & $83.0 \pm 7.7$ & $60.2 \pm 11.8$ & $5.3 \pm 4.3 \times 10^{-8}$ & $49.2 \pm 6.0$ \\
\hline I $(160)$, PMNs $(0)$ & $89.5 \pm 6.4$ & $86.4 \pm 5.0$ & $60.0 \pm 11.0$ & $5.7 \pm 2.2 \times 10^{-8}$ & $51.7 \pm 6.1$ \\
\hline I (160), PMNs (24 million) & $94.9 \pm 16.5$ & $86.3 \pm 13.4$ & $46.6 \pm 9.7$ & $5.2 \pm 2.1 \times 10^{-8}$ & $58.3 \pm 12.0$ \\
\hline
\end{tabular}

$I$, Time of ischemia in minutes; $P M N s$, Number of activated neutrophils per milliliter. $K C l$, potassium chloride; $B K N$, bradykinin; $S M$, smooth muscle. $p=$ not significant.

*Percent of total smooth muscle relaxation

†Percent of $\mathrm{KCl}(85 \mathrm{mmol} / \mathrm{L})$ contraction.

Four groups of intramyocardial arteries were compared:

Group 1: Control ring segments without ischemia or incubation with activated neutrophils

Group 2: Ring segments not subject to ischemia but incubated with activated neutrophils, 24 million per milliliter

Group 3: Ring segments after 30 minutes of ischemia, without incubation with activated neutrophils

Group 4: Ring segments after 30 minutes of ischemia and incubation with activated neutrophils, 24 million per milliliter

Baseline resting pressure and ring segment diameter for each group were compared. Three parameters were used to quantify coronary smooth muscle function. Maximum response to potassium chloride and pressure change to $85 \mathrm{mmol} / \mathrm{L}$ (epicardial arteries) or $45 \mathrm{mmol} / \mathrm{L}$ (intramyocardial arteries) concentrations of potassium chloride was used to assess smooth muscle contractility. Total smooth muscle relaxation was defined as the sum of bradykinin- and nitroprusside-induced relaxation and reported as a percentage of precontraction pressure with potassium chloride. Two parameters were used to quantify endothelial cell function. Endothelium-mediated relaxation to bradykinin was determined for each artery and expressed as a percent of total smooth muscle relaxation. $\mathrm{EC}_{50}$ (concentration producing half maximum relaxation) was used to assess endothelial sensitivity to bradykinin. By means of the Laplace relationship $(\mathrm{P}=2 \pi \mathrm{T} / \mathrm{L})$, all tension measurements were converted to pressure. Mean ( \pm standard error of mean) was calculated for each group and compared by analysis of variance. The unpaired $t$ test and rank order analysis were used to determine significant differences between groups. Values were considered to be statistically significant when $p$ was less than 0.05 . An arbitrary scoring system for semiquantitative analysis was used to quantify damage of endothelial cell surfaces in epicardial and intramyocardial arteries. Duncan's multiple rank test was used
Table III. Epicardial arteries

\begin{tabular}{lccc} 
Groups & $\begin{array}{c}\text { No. of } \\
\text { SEMs }\end{array}$ & $\begin{array}{c}\text { PMN } \\
\text { injury* }\end{array}$ & $\begin{array}{c}\text { Ischemic } \\
\text { injury }\end{array}$ \\
\hline I (0), PMNs (0) & 4 & 0.50 & 0.08 \\
I (0), PMNs (24 million) & 6 & 0.17 & 0.00 \\
I (160), PMNs (0) & 7 & 0.71 & $1.71 \dagger$ \\
I (160), PMNs (24 million) & 3 & $2.67 \dagger$ & $2.67 \dagger$ \\
\hline
\end{tabular}

$S E M S$, Scanning electron micrographs; $P M N S$, number of activated neutrophils per milliliter; $I$, time of ischemia in minutes.

*Mean injury score 1 to 4.

$\dagger p<0.05$ compared with control.

as a post test for statistical analysis of morphologic changes (statistically significant when $p$ was less than 0.05 ).

\section{Results}

\section{Epicardial arteries}

Physiology. No difference was observed in ring segment size or baseline resting pressure after the normalization procedure (Table I). Maximum contraction to potassium chloride $(125 \mathrm{mmol} / \mathrm{L})$ and contraction to an $85 \mathrm{mmol} / \mathrm{L}$ concentration of potassium chloride was not altered in the epicardial coronary arteries after ischemia with or without incubation with activated neutrophils. Endothelium-dependent relaxation to bradykinin and total smooth muscle relaxation (total relaxation to bradykinin and sodium nitroprusside) was equal between groups. A comparison of $\mathrm{EC}_{50}$, which demonstrates sensitivity of endothelial cells to bradykinin, revealed no differences among the four groups (Table II). 

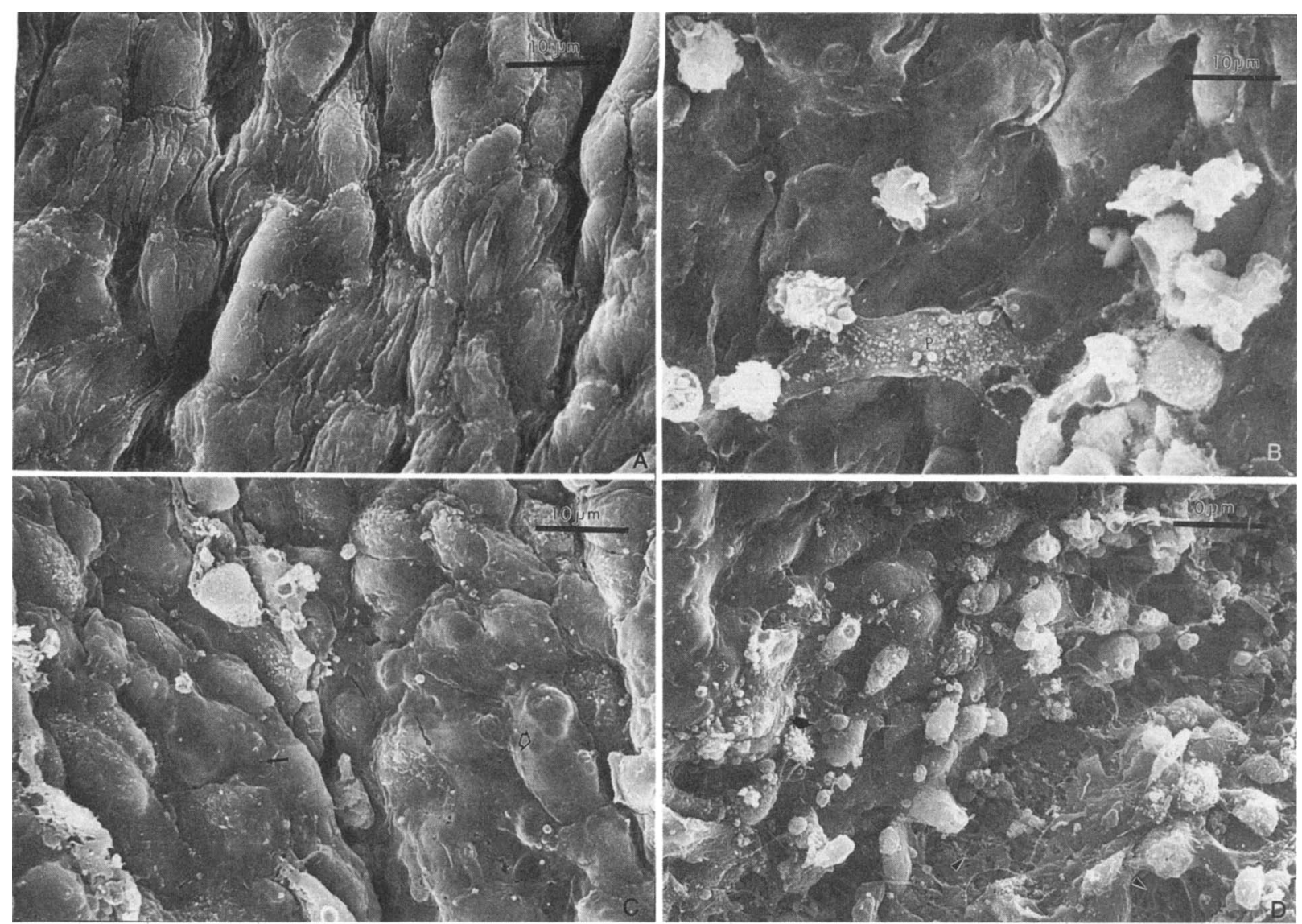

Fig. 2. Scanning electron micrographs of vascular endothelium from epicardial arteries (with and without ischemia) incubated with and without activated neutrophils (PMNs). A, Low-power micrograph of control endothelium shows a normal morphology. B, Activated neutrophils $(P)$ did little damage to the normal endothelium; only occasional holes were seen. $\mathbf{C}$, Ischemic endothelium without added polymorphonuclear leukocytes exhibited blebbing (open arrow) and small holes (black arrow), but was fairly intact. D, Ischemic endothelium exhibited extensive damage when polymorphonuclear leukocytes were added. Whole areas were denuded of endothelial cells (arrowhead); platelets and fibrin (arrow) were evident. Other areas were undamaged (plus sign) except for that caused by ischemia. (Original magnifications $\times 1200$; bar $=10 \mu \mathrm{m}$.)

Morphology. Activated neutrophils alone caused minimal damage to nonischemic large artery endothelial cells (Fig. 2, B), as compared with control endothelium (Fig. $2, A)$. After 160 minutes of ischemia, damage by activated neutrophils was moderate to severe (Fig. 2, D). The endothelial membrane beneath attached neutrophils had holes of varying sizes, giving the tissue a "moth-eaten" appearance. These holes appeared to progress to the destruction of the cell entirely, exposing the underlying connective tissue matrix. Neutrophils sometimes appeared to extravasate through holes in the endothelium. Endothelium exposed to 160 minutes of ischemia without any neutrophils (Fig. 2,C) also had evidence of damage. This included blebbing of the endothelium, bulging nuclei, the appearance of large holes, and separation of the endothelial cell borders (Table III).

\section{Intramyocardial vessels}

Physiology. No differences were observed among the four groups of myocardial arteries in ring segment diameter or baseline resting pressure (Table IV). No difference was noted in maximum contraction to a $125 \mathrm{mmol} / \mathrm{L}$ concentration of potassium among any of the four groups (Fig. 3, A). A tendency toward increased contraction to a $45 \mathrm{mmol} / \mathrm{L}$ concentration of potassium chloride after ischemia with activated neutrophils was seen $(p=0.06$; Fig. 3, $B$; Table V). Ischemia alone diminished total smooth muscle relaxation (ischemia $96.2 \% \pm 4.5 \%$, control $112.4 \% \pm 7.0 \% ; p<0.05)$; neither group was incu- 

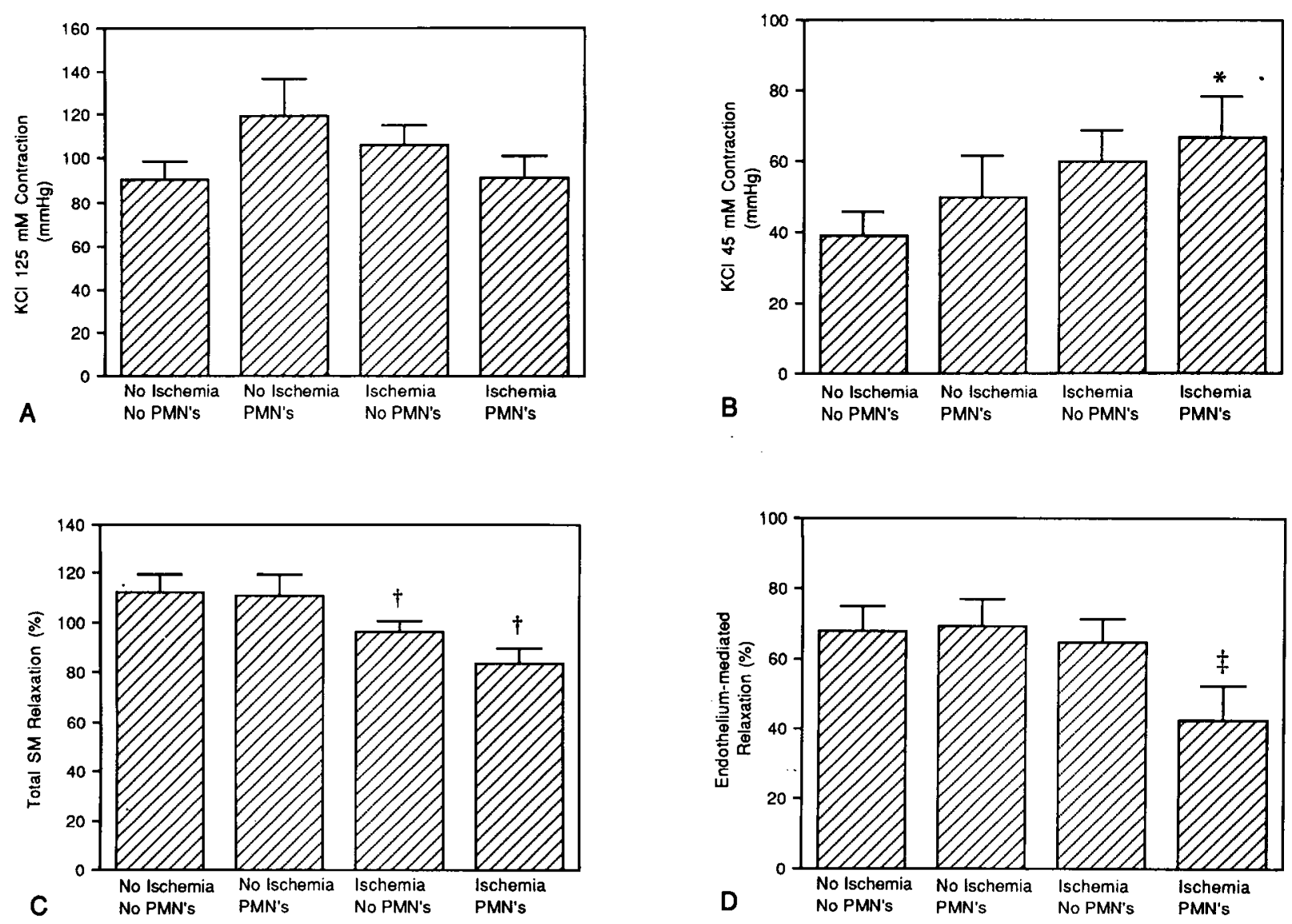

Fig. 3. Bar graphs comparing intramyocardial arteries: $\mathbf{A}$, Maximum smooth muscle $(S M)$ contraction to a 125 $\mathrm{mmol} / \mathrm{L}$ concentration of potassium $(\mathrm{KCl})$ is not significantly different in any group. B, Contraction to a $45 \mathrm{mmol} / \mathrm{L}$ concentration of $\mathrm{KCl}$ after ischemia and incubation with activated neutrophils ( $P M N$ 's) is slightly increased compared to control (no ischemia, no PMN's, $p=0.06$ ). C, Total SM relaxation after ischemia alone and after ischemia with PMN's, expressed as percent of contraction to a $45 \mathrm{mmol} / \mathrm{L}$ concentration of $\mathrm{KCl}$, is significantly decreased compared to control and after PMN's alone $(p<0.05)$. D, Endothelium-mediated relaxation, expressed as percent of maximum SM relaxation (bradykinin and sodium nitroprusside) is significantly decreased in arteries exposed to combined ischemia and PMN's $(p<0.05)$.

Table IV. Internal diameter (at $100 \mathrm{~mm} \mathrm{Hg}$ ) and normalized resting pressure of intramyocardial coronary arteries

\begin{tabular}{lccc}
\hline \multicolumn{1}{c}{ Groups } & $\begin{array}{c}\text { No. of } \\
\text { rings }\end{array}$ & ID $(\mu \mathrm{m})$ & $\begin{array}{c}\text { Baseline resting } \\
\text { pressure }(\mathrm{mm} H \mathrm{Hg})\end{array}$ \\
\hline I (0), PMNs (0) & 11 & $392.6 \pm 29.1$ & $34.6 \pm 4.4$ \\
I (0), PMNs (24 million) & 12 & $402.9 \pm 30.0$ & $33.7 \pm 3.5$ \\
I (30), PMNs (0) & 9 & $396.2 \pm 29.0$ & $32.6 \pm 3.9$ \\
I (30), PMNs (24 million) & 11 & $406.0 \pm 36.4$ & $33.5 \pm 2.6$ \\
\hline
\end{tabular}

$I D$, Internal diameter; $I$, time of ischemia in minutes; $P M N s$, number of activated neutrophils per milliliter.

bated with activated neutrophils. Ischemic arteries incubated with activated neutrophils had significantly less smooth muscle relaxation than nonischemic arteries incubated with neutrophils (nonischemic arteries $110.6 \% \pm 8.9 \%$ versus ischemic vessels $84.2 \% \pm 5.5 \%$, $p<0.05)$. Activated neutrophils added after ischemia had no greater effect than ischemia alone on total smooth muscle relaxation to sodium nitroprusside (96.2\% $\pm 4.5 \%$ after 30 minutes of ischemia without neutrophils versus $84.2 \% \pm 5.5 \%$ after ischemia and incubation 


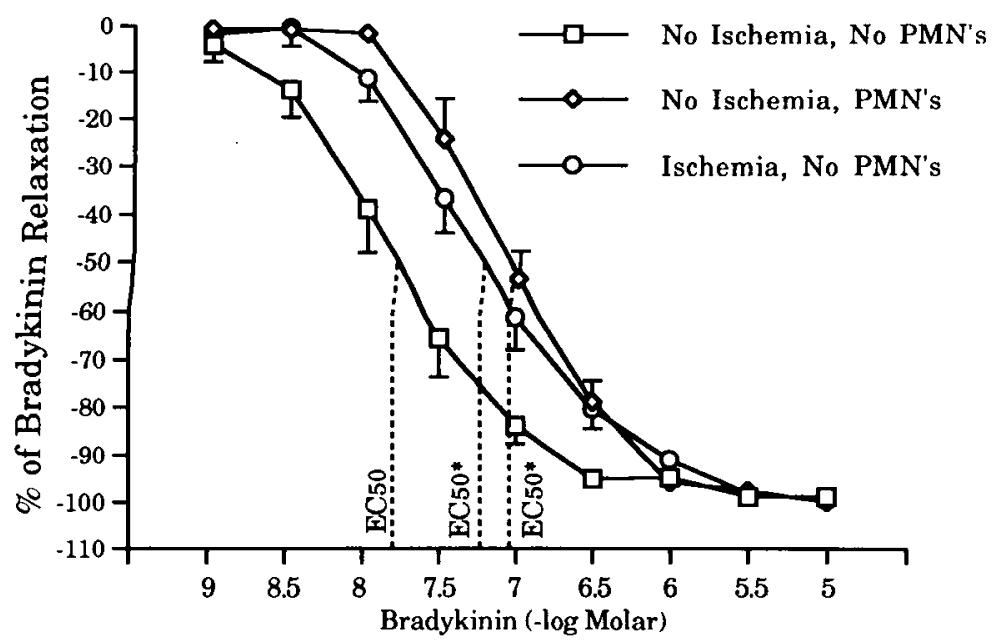

Fig. 4. Cumulative concentration response curves to bradykinin in control ring segments (no ischemia, no activated neutrophils [PMN's]) (square), ring segments after incubation with PMN's (diamond), and ring segments after 30 minutes of ischemia and without PMN's (circle). Values are shown as mean bradykinin relaxation \pm standard error of the mean. Concentration response curves and $\mathrm{EC}_{50}$ (concentration of bradykinin producing $50 \%$ of maximum endothelium-mediated relaxation) after ischemia and after ischemia followed by incubation with PMN's is shifted to the right $(p<0.05)$. $\mathrm{EC}_{50}$ for intramyocardial arteries incubated with PMN's after 30 minutes of ischemia could not be calculated because most ring segments did not respond to bradykinin. This demonstrates maximum injury in intramyocardial artery endothelium after combined ischemia and incubation with neutrophils.

Table V. Reactivity of intramyocardial coronary arteries

\begin{tabular}{lccccc}
\hline \multicolumn{1}{c}{ Groups } & $\begin{array}{c}K C l(125 \mathrm{mmol} / \mathrm{L}) \\
(\mathrm{mm} \mathrm{Hg})\end{array}$ & $\begin{array}{c}K C l(45 \mathrm{mmol} / \mathrm{L}) \\
(\mathrm{mm} \mathrm{Hg})\end{array}$ & $\begin{array}{c}\text { Relaxation } \\
\text { to } B K N^{*}(\%)\end{array}$ & $\begin{array}{c}E C_{50} \text { BKN } \\
(\text { mmol/L) }\end{array}$ & $\begin{array}{c}\text { Total SM } \\
\text { relaxation }(\%)\end{array}$ \\
\hline I (0), PMNs (0) & $90.0 \pm 9.1$ & $39.1 \pm 6.6$ & $67.7 \pm 7.0$ & $2.5 \pm 2.2 \times 10^{-8}$ & $112.4 \pm 7$ \\
I (0), PMNs (24 million) & $119.9 \pm 19.3$ & $49.7 \pm 12.0$ & $69.0 \pm 7.9$ & $10.0 \pm 2.2 \times 10^{-8} \star$ & $110.6 \pm 8.9$ \\
I (30), PMNs (0) & $106.1 \pm 9.3$ & $60.0 \pm 8.8$ & $64.7 \pm 6.8$ & $9.2 \pm 2.4 \times 10^{-8} \star$ & $96.2 \pm 4.5 \S$ \\
I (30), PMNs (24 million) & $90.9 \pm 11.0$ & $66.6 \pm 12.0 \ddagger$ & $42.7 \pm 9.4 \S$ & $\mathbb{I}$ & $84.2 \pm 5.5$ \\
\hline
\end{tabular}

$I$, Time of ischemia in minutes; $P M N s$, number of activated neutrophils per milliliter.

*Percent of total smooth muscle $(S M)$ relaxation.

$\dagger$ Percent of $\mathrm{KCl}(45 \mathrm{mmol} / \mathrm{L})$ contraction.

$\ddagger p=0.06$ compared to I $(0)$, PMNs $(0)$.

$\$ p<0.05$ compared to I ( 0 ), PMNs ( 0 ).

IEC $_{50}$ not calculated because of no relaxation to bradykinin $(B K N)$.

$\| p<0.05$ compared to I (0), PMNs $(0)$ and to I (0), PMNs (24 million).

with neutrophils; $p=0.06$; Fig. $3, C$ ). Maximum endothelium-dependent relaxation in rings without ischemia or neutrophils, rings without ischemia and with neutrophils, and rings with ischemia and no neutrophils were not different (Fig. 3, D). However, endothelium-dependent relaxation was significantly decreased after combined ischemia and incubation with activated neutrophils compared to control values $(42.7 \% \pm 9.4 \%$ versus $67.7 \%$ $\pm 7.0 \%)$. Sensitivity to bradykinin $\left(\mathrm{EC}_{50}\right)$ was decreased after both activated neutrophils alone, group 2 $\left(9.2 \pm 2.4 \times 10^{-8}\right)$, and ischemia alone, group $3(10 \pm$ $\left.2.2 \times 10^{-8}\right)$, compared with control $\left(2.5 \pm 2.2 \times 10^{-8}\right)$. $\mathrm{EC}_{50}$ could not be calculated for several ring segments subjected to 30 minutes of ischemia and incubation with activated neutrophils because bradykinin elicited no relaxation. This comparison, therefore, could not be made (Fig. 4, Table V) and demonstrates maximum injury to endothelial cells.

Morphology. Ultrastructural results in the intramyocardial artery endothelium were similar to those in epicardial arteries. That is, without ischemia, neutrophils did little or no damage to the endothelium (Fig. 5, $B$ ) compared with the control endothelium (Fig. 5, A). However, after 30 minutes of ischemia, damage by activated neutrophils was moderate to severe (Fig. 5, D). As with the larger arteries, ischemic damage was also seen in 

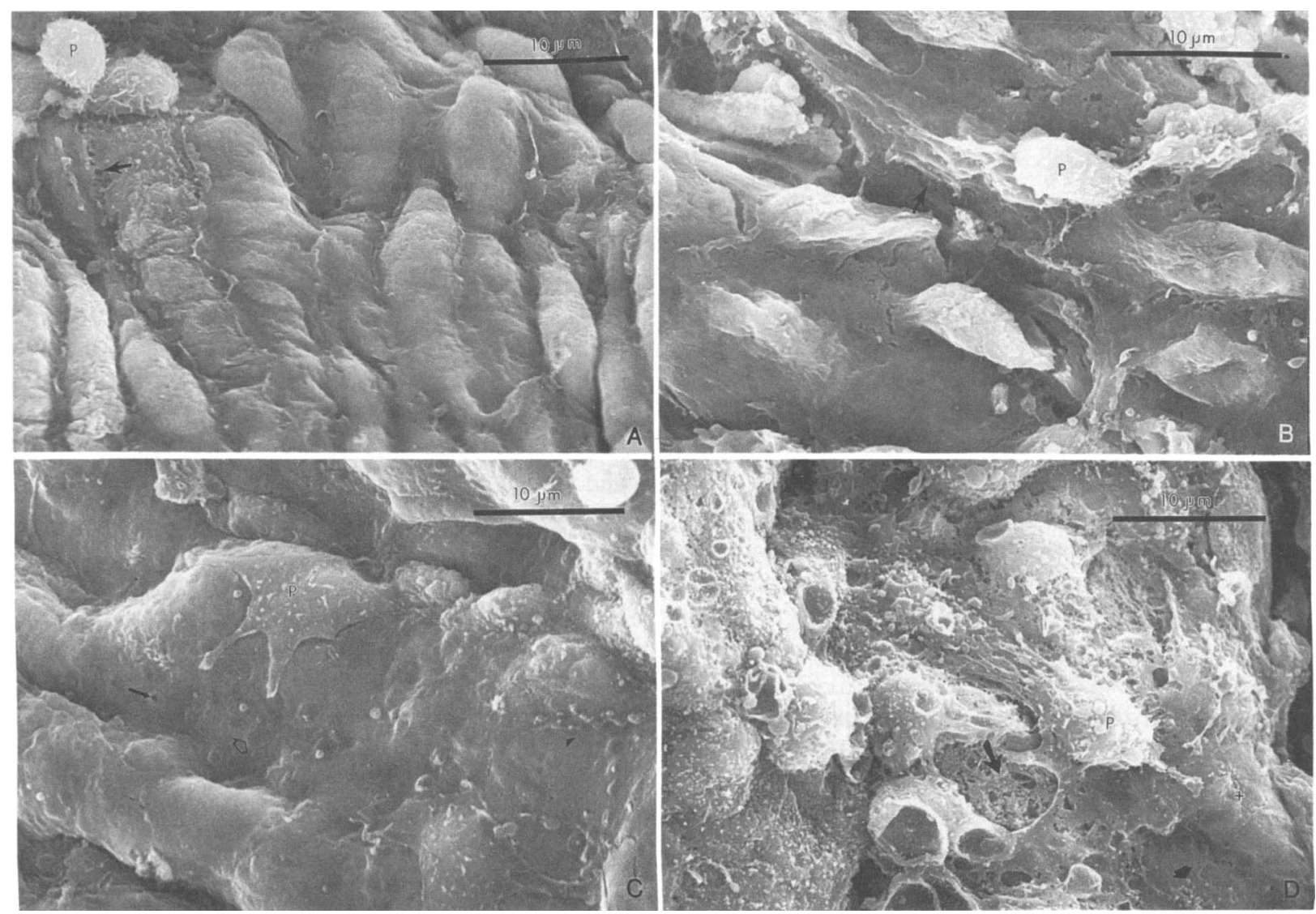

Fig. 5. Scanning electron micrographs of intramyocardial arterial endothelium (with and without ischemia) incubated with and without activated neutrophils (PMNs). A, Control endothelium exhibited normal morphology, with an occasional PMN $(P)$, associated with some endothelial damage (arrow). B, Activated neutrophils $(P)$ did little damage to the normal endothelium; only occasional holes were seen (arrow). C, Ischemic endothelium without added PMNs exhibited blebbing (open arrow) and small holes (small arrow), but was fairly intact. D, Ischemic endothelium exhibited extensive damage when PMNs were added. Damage appears "lacy" in the first stages; eventually nothing recognizable is left of the endothelial cell (long arrow). Other areas appear undamaged (plus sign) and platelets (short arrow) and fibrin are evident in abundance. All micrographs are of the same magnification; bar $=10 \mu \mathrm{m}$.

intramyocardial arteries subject to 30 minutes of ischemia without added neutrophils (Fig. 5, $C$, Table VI).

\section{Discussion}

This study supports the hypothesis that activated neutrophils magnify damage to intramyocardial artery endothelial cells and may be important in reperfusion injury. In vivo studies of microvasculature have clearly demonstrated that ischemia-reperfusion promotes the adherence of leukocytes to vascular endothelium. ${ }^{14-16}$ Other studies have shown that injury to coronary microvascular endothelium, as reflected in loss of its barrier function, is due to the effect of neutrophils. ${ }^{13}$

There were five major findings of this study:

First, a major decrease in intramyocardial artery function occurred after ischemia and storage with neutrophils and no diminution of function in large epicardial arteries even though time of ischemia was much longer. We can only speculate as to reasons for differing functional response to injury between large epicardial and small intramyocardial arteries. Functional responses may be the consequence of the influence of local tissue injury and inflammatory mediators that promote microvascular and small artery injury. Thicker walls of epicardial arteries may also be protective from the influence of neighboring ischemic tissue that may elaborate mediators that promote expression of endothelial adhesion molecules.

The second finding is that ischemia alone decreased smooth muscle relaxation in intramyocardial arteries. Addition of neutrophils did not further decrease total 
Table VI. Intramyocardial arteries

\begin{tabular}{lcll}
\hline \multicolumn{1}{c}{ Groups } & $\begin{array}{c}\text { No. of } \\
\text { SEMs }\end{array}$ & $\begin{array}{c}\text { PMN } \\
\text { injury* }\end{array}$ & $\begin{array}{c}\text { Ischemic } \\
\text { injury }\end{array}$ \\
\hline I (0), PMNs (0) & $n=5$ & 0.00 & 0.30 \\
I (0), PMNs (24 million) & $n=3$ & 0.33 & 0.00 \\
I (160), PMNs (0) & $n=5$ & 0.30 & $1.20 \dagger$ \\
I (160), PMNs (24 million) & $n=3$ & $1.66 \dagger$ & $2.50 \dagger$ \\
\hline
\end{tabular}

$S E M s$, Scanning electron micrographs; $I$, time of ischemia in minutes; $P M N s$, number of activated neutrophils per milliliter.

*Mean injury score 1 to 4 .

$\dagger p<0.05$.

relaxation. In this study, under control conditions total relaxation in control ring segments was twice as great in intramyocardial as in epicardial arteries.

The third finding is increased contraction after the combination of ischemia and activated neutrophils in intramyocardial arteries. Presumably ischemia and storage with neutrophils created endothelial dysfunction and decreased EDRF production. Decrease of basal EDRF resulted in increased contraction.

The fourth finding is a subtle decrease in endotheliummediated relaxation in response to bradykinin, which was observed after ischemia alone and after storage with neutrophils alone and manifest by a shift in $\mathrm{EC}_{50}$. This latter finding may reflect minor endothelial dysfunction associated with cold, oxygenated storage. However, a significant decrease in peak response to bradykinin was found only after ischemia combined with incubation with neutrophils. At least two possible mechanisms for major impairment of endothelium-dependent smooth muscle relaxation to bradykinin exist. First, intramyocardial artery smooth muscle cells may lose ability to respond to EDRF. Second, endothelial cell vasodilator function may diminish. We found diminished endothelium-mediated vasodilatation in addition to diminished total smooth muscle vascular response, which suggests that both mechanisms are operative.

Impaired sensitivity to bradykinin occurred in the absence of neutrophils after ischemia in intramyocardial arteries. Continuous production of oxygen-derived free radicals by endothelial cells themselves cannot be excluded, nor can endothelial cell damage by oxygen-derived free radicals produced by native neutrophils remaining in arteries during the ischemic period. Filling with nonactivated neutrophils and incubation with oxygenated PSS at $37^{\circ} \mathrm{C}$ did not cause alteration of endothelial cell function (data not shown).

Finally, in contrast to the functional difference between epicardial and intramyocardial arteries, we found comparable damage by electron microscopy. We can only speculate as to the differences between epicardial artery morphology and function. One hypothesis is that there are greatly differing sensitivities to vasodilator stimuli between the large and small arteries and that endothelial injury may have less profound physiologic consequences. Endothelial cells of epicardial arteries probably have different characteristics from endothelial cells of intramyocardial arteries. ${ }^{17}$ Persistence of epicardial endothelial function after 160 minutes of ischemia and incubation with neutrophils may be explained by differences in endothelial cell and EDRF sensitivity to injury. Endothelial cells are highly differentiated cells, covering the entire surface of the vascular system. Endothelial cells secrete numerous chemicals, including anticoagulant (prostaglandin) and procoagulant substances (thromboxane) and vasodilator (EDRF) and vasoconstrictor (endothelin) substances. Arteries from different vascular beds demonstrate different responses to vasoactive substances. Han and colleagues ${ }^{18}$ demonstrated significant differences in endothelin and EDRF production in response to tumor necrosis factor between cultured pulmonary artery and aortic endothelial cells. It is probable, therefore, that endothelium from epicardial arteries has different functions and sensitivities than endothelial cells covering intramyocardial arteries. For example, Meyrick, Christman, and Jasmok ${ }^{19}$ showed that endothelial cells cultivated from pulmonary microvessels are more resistant to tumor necrosis factor than cells cultivated from large pulmonary arteries.

We found a significant decrease in tolerance to ischemia and activated neutrophils in intramyocardial arteries. Whereas significant histologic alterations were found in both epicardial and intramyocardial arteries as a result of ischemia and neutrophil injury, decreased function was found only in intramyocardial endothelium.

Clinical implications. Myocardial function is not salvaged after 160 minutes of ischemia with reperfusion. We demonstrated normal endothelial function of epicardial arteries even after 160 minutes of ischemia combined with neutrophils. Intramyocardial but not epicardial artery endothelium may, therefore, contribute to or influence ischemia and reperfusion injury of the heart.

In the clinical situation, flow through epicardial arteries is possibly not limited by ischemia or neutrophil activation. Intramyocardial arteries and arterioles, however, may inhibit reperfusion because EDRF-mediated relaxation is significantly decreased. EDRF not only relaxes vascular smooth muscle but also inhibits platelet adhesion and is itself a potent antiaggregatory substance. ${ }^{20}$ These data suggest that increased numbers of activated neutrophils observed after cardiopulmonary bypass, percutaneous transluminal balloon angioplasty, ${ }^{21}$ and thrombolytic therapy may be involved in triggering and enhancing 
endothelial cell damage and could play a key role in microvascular vasospasm and myocardial injury. This is particularly true when the ischemia-reperfusion injury is severe enough to cause local tissue injury. This observation has been supported by studies demonstrating amelioration of reperfusion injury by the use of leukocyte filters. $^{6}$

In vivo studies confirm vascular injury by abnormalities of reperfusion but do not identify the vascular locus targeted by the combination of ischemia and activated neutrophils. These in vitro studies allowed evaluation of the coronary vasculature without the confounding effects of in vivo reperfusion edema and myocellular contracture.

\section{REFER E N C ES}

1. Furchgott RF, Zawadzki JV. The obligatory role of endothelial cells in the relaxation of arterial smooth muscle by acetylcholine. Nature 1980;288:373-6.

2. Furchgott RF, Vanhoutte PM. Endothelium derived relaxing and contracting factors. FASEB J 1989;3:200718.

3. Ku DD. Coronary vasculature reactivity after acute myocardial ischemia. Science 1982;218:576-8.

4. Ignarro LJ. Biological actions and properties of endothelium-derived nitric oxide formed and released from artery and vein. Circ Res 1989;65:1-21.

5. Tsao PhS, Aoki N, Lefer DJ, Johnson G III, Lefer AM. Time course of endothelial dysfunction and myocardial injury during myocardial ischemia and reperfusion in the cat. Circulation 1990;82:1402-6.

6. Litt MR, Jeremy RW, Weisman HF, Winkelstein JA, Becker LC. Neutrophil depletion limited to reperfusion reduced myocardial infarct size after 90 minutes of ischemia: evidence for neutrophil-mediated reperfusion injury. Circulation 1989;80:1816-27.

7. Quillen JE, Sellke F, Brooks LA, Harrison DG. Ischemiareperfusion impairs endothelial-dependent relaxation of coronary microvessels but does not affect large arteries. Circulation 1990;82:586-94.

8. Dignan JR, Dyke MC, Abd-Elfattah AS, et al. Coronary artery endothelial cell and global myocardial ischemia. Ann Thorac Surg 1992;53:311-7.

9. Henson PM. The immunologic release of constituents from neutrophil leukocytes: the role of antibody and complement on nonphagocytosable surfaces or phagocytosable particles. J Immunol 1971;107:1535-46.

10. Fletcher MP, Stahl GL, Longhurst JC. In vivo and in vitro assessment of porcine neutrophil activation responses to chemoattractants: flow cytometric evidence for the selective absence of formyl peptide receptors. J Leukocozyte Biol 1990;47:355-65.

11. Webster RO, Wysolmerski RB, Lagunoff D. Enhancement of human polymorphonuclear leukocyte adherence to plastic and endothelium by phorbol myristate acetate: comparison with human C5a. Am J Pathol 1986;125:369-78.

12. Dignan RJ, Yey T Jr, Dyke CM, et al. Reactivity of gastroepiploic and internal mammary arteries: relevance to coronary artery bypass grafting. J THORAC CARDIOVASC SURG 1992;103:116-23.

13. Sheridan FM, Dauber IM, McMurtry IF, Lesnefsky EJ, Horwitz LD. Role of leukocytes in coronary vascular endothelial injury due to ischemia and reperfusion. Circ Res 1991;69:1566-74.

14. Granger DN, Benoit JN, Suzuki M, Grisham MB. Leucocyte adherence to venular endothelium during ischemia reperfusion. Am J Physiol 1989;257:G683-8.

15. Messmer K, Sack FU, Menger MD, Bartlett R, Barker JH, Hammersen F. White cell-endothelium interaction during postischemic reperfusion of skin and skeletal muscle. Adv Exp Med Biol 1988;242:95-8.

16. Zeintl H, Sack FU, Intaglietta M, Messmer K. Computer assisted leukocyte adhesion measurement in intravital microscopy. Int J Microcirc Clin Exp 1989;8:293-302.

17. Furchgott RF. Role of endothelium in response of vascular smooth muscle. Circ Res 1983;53:557-73.

18. Han JJ, Bechard DE, Fisher BJ, Walsh CJ, Drenning D, Fowler AA. Tumor necrosis factor indices: differential production of endothelium derived relaxing factor in cultured porcine aortic and pulmonary endothelial cells. Am Rev Respir Dis 1992;145:A242.

19. Meyrick B, Christman B, Jasmok G. Effect of recombinant tumor necrosis factor-alpha on cultured pulmonary artery and lung microvascular endothelial monolayer. Am J Pathol 1991;138:93-101.

20. Cohen RA, Shephard JT, Vanhoutte PM. Inhibitory role of the endothelium in response of isolated coronary artery to platelets. Science 1983;221:273-4.

21. De Servi S, Mazzone A, Ricevuti G, et al. Granulocyte activation after coronary angioplasty in humans. Circulation 1990;82:140-6. 